\title{
Approximate Solution for the Thin Film Flow Problem of a Third Grade Fluid Using Spline Collocation Method
}

\author{
Hetal Shah ${ }^{1}$, Jigisha Pandya ${ }^{2}, \quad$ Pinky Shah ${ }^{1}$ \\ ${ }^{1}$ Research Scholar, Mathematics Department, Veer Narmad South Gujarat University, Surat, India. \\ ${ }^{2}$ Assisant Professor, Department of Mathematics, Sarvajanik College of Engineering \& Technology, Surat, India. \\ Email: jigisha.pandya@scet.ac.in
}

\begin{abstract}
This paper aims to present the approximate solution for a thin film flow of a third grade fluid down an inclined plane. The variation of the velocity field for different parameters has been analyzed. The spline collocation method is used to obtain the accurate solution. The results are shown in a tabular form as well as in a graphical manner.
\end{abstract}

Keywords: Spline collocation method, Thin film flow, Third grade fluid, Inclined plane, Nonlinear boundary value problem

\section{Introduction}

In recent years, the research in flows of non-Newtonian fluids has been intensified due to their wide application in industry and engineering, especially with the emergence of polymer industry, while dealing with the non-Newtonian fluids is of great challenge in solution of governing non-linear differential equations. Many Numerical and analytical techniques have been developed to solve nonlinear differential equations by many researchers. These methods include the artificial parameter methods by $\mathrm{He}$ [1], the variational iteration method by He [2], the homotopy analysis method by Liao [3], the optimal homotopy asymptotic method (OHAM) by Marinca and Herisanu [4], the optimum homotopy asymptotic method by Mabood [5], an implicate finite difference method by Itishree et al [6], the spline collocation method [7], etc.

In this paper, we considered the steady uni-dimensional flow of an incompressible third grade fluid down a uniform inclined plane, for the third grade fluid, the first four terms of Taylor series are using the stress rate of strain relation. The grade fluid models are complicated due to a large number of physical parameters that have to be determined experimentally. Many numerical techniques have been purposed by various researchers. An efficient approximate numerical solution will find enormous applications. In this paper we have solved the governing non-linear differential equation of the problem using cubic spline collocation method for various parameters.

This paper is organized as follows: in section 2, governing equation of the problem is presented, basic principles of spline collocation iteration method are explained in section 3 and the solutions are given in section 4 . In section 5 , we analyse the results by comparing the solution with the available results.

\section{Governing Equation}

The thin film flow of a third grade fluid down an inclined plane of inclination $\alpha \neq 0$ is governed by the following nonlinear boundary value problem $[5]$

$$
\begin{gathered}
\frac{d^{2} u}{d y^{2}}+\frac{6\left(\beta_{2}+\beta_{3}\right)}{\mu}\left(\frac{d u}{d y}\right)^{2} \frac{d^{2} u}{d y^{2}}+\frac{\rho g \sin \alpha}{\mu}=0 \\
u(0)=0, \quad \mathrm{u}^{\prime}(\delta)=0
\end{gathered}
$$

Introducing the parameters

$$
y=\delta y^{*}, u=\frac{\delta^{2} \rho g \sin \alpha}{\mu} u^{*}, \beta^{*}=\frac{3 \delta^{2} \rho^{2} g^{2} \sin ^{2} \alpha}{\mu^{3}}\left(\beta_{2}+\beta_{3}\right)
$$


where $\mu$ is the dynamic viscosity, $\rho$ is the acceleration due to the gravity, $\delta$ is the fluid density and $\beta>0$ is the material constant of a third grade fluid, $\bar{x}$ and $\bar{y}$ are the directions, and $\bar{u}$ and $\bar{v}$ are the velocity components in these directions respectively, $\mathrm{g}_{1}$ is gravitational constant acting in the downward direction.

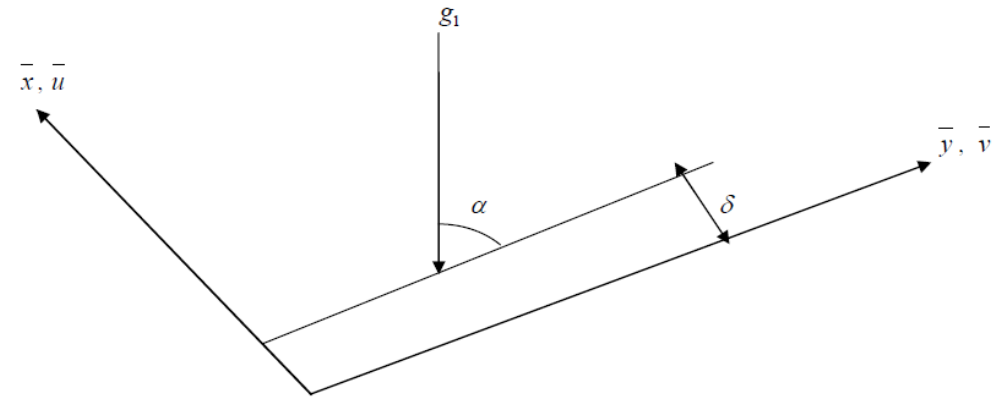

Figure 1. The geometry of the problem

The problem in Equation (1) and (2), after omitting asterisks, takes the following form

$$
\begin{gathered}
\frac{d^{2} \phi}{d y^{2}}+6 \beta\left(\frac{d \phi}{d y}\right)^{2} \frac{d^{2} \phi}{d y^{2}}+m=0 \\
\phi(0)=0, \phi^{\prime}(1)=0
\end{gathered}
$$

We note that Equation (4) is a second order nonlinear differential equation with two boundary conditions; therefore, it is a well-posed problem.

\section{Basic Principles of Spline Collocation}

The use of spline functions with moments for the solution of nonlinear differential equation was suggested by Blue [8]. Let us consider a linear two-point boundary value problem

$$
y^{\prime \prime}(x)+p(x) y^{\prime}(x)+q(x) y(x)=r(x)
$$

subject to the boundary conditions

$$
\begin{array}{ll}
G_{1}\left[y(a), y^{\prime}(a)\right]=0 & \text { at } x=a \\
G_{2}\left[y(b), y^{\prime}(b)\right]=0 & \text { at } x=b
\end{array}
$$

Since $s(x)$ is a cubic spline interpolating $y(x)$ given by equation (6), we have $\mathrm{s}\left(x_{i}\right)=y\left(x_{i}\right)$, and also $s^{\prime \prime}(x)$ is a linear function. Let us define this $s^{\prime \prime}(x)$ in the subinterval $\left[x_{i}, x_{i}+1\right]$ of $[a, b]$ as follows:

$$
s^{\prime \prime}(x)=y_{i+1}^{\prime \prime} \frac{x-x_{i}}{h_{i+1}}+y_{i}^{\prime \prime} \frac{x_{i+1}-x}{h_{i+1}}, \quad i=0,1,2, \ldots . n-1
$$

where $h_{i+1}=x_{i+1}-x_{i}$

Two successive integrations produce a cubic spline $(x)$ in $\left[\mathrm{x}_{\mathrm{i}+1}, \mathrm{x}_{\mathrm{i}}\right]$ which has the form

$$
s(x)=y_{i+1}^{\prime \prime} \frac{\left(x-x_{i}\right)^{3}}{6 h_{i+1}}+y_{i}^{\prime \prime} \frac{\left(x_{i+1}-x\right)^{3}}{6 h_{i+1}}+\frac{A\left(x_{i+1}-x\right)}{h_{i+1}}+\frac{B\left(x-x_{i}\right)}{h_{i+1}}
$$

with $\mathrm{A}$ and $\mathrm{B}$ being constants to be determined. Interpolation condition provides their evaluation.

Equation (10) is given due to these constants as follows: 


$$
\begin{array}{r}
s(x)=y_{i+1}^{\prime \prime} \frac{\left(x-x_{i}\right)^{3}}{6 h_{i+1}}+y_{i}^{\prime \prime} \frac{\left(x_{i+1}-x\right)^{3}}{6 h_{i+1}}+\left(y_{i}-\frac{h_{i+1}^{2}}{6} y_{i}^{\prime \prime}\right) \\
\frac{\left(x_{i+1}-x\right)}{h_{i+1}}+\left(y_{i+1}-\frac{h_{i}^{2}}{6} y_{i+1}^{\prime \prime}\right) \frac{\left(x-x_{i}\right)}{h_{i+1}}
\end{array}
$$

Similarly, $s(x)$ can be obtained in the interval $\left[\mathrm{x}_{\mathrm{i}-1}, \mathrm{x}_{\mathrm{i}}\right]$ as

$$
\begin{aligned}
& s(x)=y_{i}^{\prime \prime} \frac{\left(x-x_{i-1}\right)^{3}}{6 h_{i}}+y_{i-1}^{\prime \prime} \frac{\left(x_{i}-x\right)^{3}}{6 h_{i}}+\left(y_{i}-\frac{h_{i}^{2}}{6} y_{i}^{\prime \prime}\right) \frac{\left(x-x_{i-1}\right)}{h_{i}}+ \\
& \left(y_{i-1}-\frac{h_{i}^{2}}{6} y_{i-1}^{\prime \prime}\right) \frac{\left(x_{i}-x\right)}{h_{i}}
\end{aligned}
$$

Deriving $s^{\prime}(x)$ at $x=x_{i}$ from the equation (12) which is denoted by $s^{\prime}\left(x_{i+}\right)$, we have

$$
s^{\prime}\left(x_{i+}\right)=-\frac{h_{i+1}}{3} y_{i}^{\prime \prime}-\frac{h_{i+1}}{6} y_{i+1}^{\prime \prime}+\frac{y_{i+1}-y_{i}}{h_{i+1}}, \quad i=0,1,2, \ldots . n-1
$$

and in the same way

$$
s^{\prime}\left(x_{i-}\right)=\frac{h_{i}}{6} y_{i-1}^{\prime \prime}-\frac{h_{i}}{3} y_{i}^{\prime \prime}+\frac{y_{i}-y_{i-1}}{h_{i}}, \quad i=1,2, \ldots . n
$$

Continuity of $s^{\prime \prime}(x)$ at $x=x_{i}$ requires that $s^{\prime \prime}\left(x_{i+}\right)=s^{\prime \prime}\left(x_{i-}\right)$ so that

$$
h_{i} y_{i+1}-\left(h_{i}+h_{i+1}\right) y_{i}+h_{i+1} y_{i-1}=h_{i} h_{i+1}\left(\frac{h_{i}}{6} y_{i-1}^{\prime \prime}+\frac{h_{i}+h_{i+1}}{6} y_{i}^{\prime \prime}+\frac{h_{i+1}}{6} y_{i+1}^{\prime \prime}\right), i=1,2, \ldots . n-1
$$

This equation gives a system of $(\mathrm{n}-1)$ equations with $(\mathrm{n}+1)$ variables $y_{i}, i=0,1,2, \ldots . n$ to be determined. Therefore, the moments $y_{i}^{\prime \prime}, i=0,1,2, \ldots . . n$ can be obtained from relation (12) if a curve is initially fitted to the data and two extra conditions are considered to complete the system.

Here our objective is to solve the differential equation (6) with the help of relation (15). Let us express the equation (6) in the form

$$
y^{\prime \prime}(x)=f\left(x, y, y^{\prime}\right)
$$

subject to the boundary conditions (7) and (8). From these boundary conditions, it is seen that there are four pairs of boundary conditions as possible combinations viz.

(i) $\quad y(a)=K \quad ; \quad y(b)=L$

(ii) $\quad y(a)=K \quad ; \quad y^{\prime}(b)=L$

(iii) $\quad y^{\prime}(a)=K \quad ; \quad y(b)=L$

(iv) $\quad y^{\prime}(a)=K \quad ; \quad y^{\prime}(b)=L$

The relation (15) will assume the form for case (i) as

$$
\begin{aligned}
h_{1} y_{2}- & \left(h_{1}+h_{2}\right) y_{1}+h_{2} y_{0}=\frac{h_{1} h_{2}}{6}\left(\frac{h_{1}}{6} y_{0}^{\prime \prime}+\frac{h_{1}+h_{2}}{3} y_{1}^{\prime \prime}+\frac{h_{2}}{6} y_{2}^{\prime \prime}\right) \\
h_{i} y_{i+1}- & \left(h_{i}+h_{i+1}\right) y_{i}+h_{i+1} y_{i-1}= \\
& \quad \frac{h_{i} h_{i+1}}{6}\left(\frac{h_{i}}{6} y_{i-1}^{\prime \prime}+\frac{h_{i}+h_{i+1}}{3} y_{i}^{\prime \prime}+\frac{h_{i+1}}{6} y_{i+1}^{\prime \prime}\right), i=1,2,3, \ldots . n-1 \\
h_{n-1} y_{n}- & \left(h_{n}+h_{n-1}\right) y_{n-1}+h_{n} y_{n-2}= \\
& \quad \frac{h_{n} h_{n+1}}{6}\left(\frac{h_{n}}{6} y_{n-2}^{\prime \prime}+\frac{h_{n-1}+h_{n}}{3} y_{n-1}^{\prime \prime}+\frac{h_{n-1}}{6} y_{n}^{\prime \prime}\right)
\end{aligned}
$$

Case (ii) with equation (15) gives 


$$
\begin{aligned}
& -2 y_{1}+y_{2}=\frac{h_{1}^{2}}{6}\left(y_{0}^{\prime \prime}+4 y_{1}^{\prime \prime}+y_{2}^{\prime \prime}\right)-y_{0} \\
& h_{i} y_{i+1}-\left(h_{i}+h_{i+1}\right) y_{i}+h_{i+1} y_{i-1}= \\
& \quad \frac{h_{i} h_{i+1}}{6}\left(\frac{h_{i}}{6} y_{i-1}^{\prime \prime}+\frac{h_{i}+h_{i+1}}{3} y_{i}^{\prime \prime}+\frac{h_{i+1}}{6} y_{i+1}^{\prime \prime}\right), i=1,2,3, \ldots . n-1 \\
& y_{n-1}-y_{n}=\frac{h_{n}^{2}}{6}\left(y_{n-1}^{\prime \prime}+2 y_{n}^{\prime \prime}\right)+h_{n} y_{n}^{\prime}
\end{aligned}
$$

Similarly, we are able to deal with remaining cases. It is observed for any case that on the left hand side the equations are obtained for which the coefficient matrix is an upper tridiagonal one.

Now in order to obtain a solution to equation (6) with the boundary conditions given in equations (7) and (8), we fit a straight line $y(x)=m x+c$ through the boundary points, which is of course an initial guess, and the moments $y_{i}^{\prime \prime}$ are calculated from the relation (12) at the nodal points $x=x_{i}$ as

$$
y_{i}^{\prime \prime}=f\left(x, y_{i}, y_{i}^{\prime}\right) \text { for } i=0,1,2 \ldots n
$$

These moments are now utilized to evaluate $y_{i}, \mathrm{i}=0,1,2 \ldots \mathrm{n}$, through the relation (16) along with the two additional equations from boundary conditions. This can be finished by solving merely a tridiagonal system of equations. The results so obtained can again be improved by continuing the same process till the desired solutions are found or two successive iterations produce the same values.

\section{Solution by Spline Collocation Iteration Method}

In order to find out the approximation $s(y)$ of $\phi(y)$ described in equation (20) satisfying the boundary conditions (21), a line $\phi(y)=a y+b$ is assumed to be the first approximation to start with the iterative scheme. The straight line $\phi(y)=0$ can be fitted through these points $\mathrm{y}=0$ and $\mathrm{y}=1$. The calculation of $\phi_{i}, \mathrm{i}=0,1,2 \ldots \mathrm{N}$ is to be carried out through the solution of tridiagonal system of $(N+1)$ equations as explained in the above method.

$$
\begin{gathered}
-2 \phi_{1}+\phi_{2}=\frac{h^{2}}{6}\left[\phi_{0}^{\prime \prime}+4 \phi_{1}^{\prime \prime}+\phi_{2}^{\prime \prime}\right] \\
\phi_{i-1}-2 \phi_{i}+\phi_{i+1}=\frac{h^{2}}{6}\left[\phi_{i-1}^{\prime \prime}+4 \phi_{i}^{\prime \prime}+\phi_{i+1}^{\prime \prime}\right], \quad i=1,2,3, \ldots N-1 \\
\phi_{N-1}-\phi_{N}=\frac{h^{2}}{6}\left(\phi_{N-1}^{\prime \prime}+2 \phi_{N}^{\prime \prime}\right)
\end{gathered}
$$

Here $\phi_{i}^{\prime \prime}=\frac{-m}{\left(1+6 \beta \phi_{i}^{2}\right)}$, in which $\phi_{i}$ and $\phi_{i}^{\prime}$ can be found from the equation of straight line $\phi(y)=0$ and $\phi^{\prime}(y)=0$, respectively as an initial guess to start with the procedure. That is the first iteration, and so on.

The results after obtaining the solution of above tridiagonal system are given in the table below. Comparisons are performed between the spline collocation method, the homotopy perturbation method (HPM), optimal homotopy asymptotic method (OHAM) and the numerical results from the Runge Kutta Fehlberg four fifth orders method (NM). 
Table 1. Comparison of numerical solution of spline collocation with OHAM, NM and HPM for $\beta=1.4, \mathrm{~m}=0.75$

\begin{tabular}{|c|c|c|c|c|}
\hline $\begin{array}{c}\text { Values' } \\
\text { of } \mathbf{y}\end{array}$ & $\begin{array}{c}\text { Solution through } \\
\text { spline collocation } \\
\text { method }\end{array}$ & $\begin{array}{c}\text { Solution } \\
\text { through OHAM }\end{array}$ & $\begin{array}{c}\text { Solution } \\
\text { through } \\
\text { NM }\end{array}$ & $\begin{array}{c}\text { Solution } \\
\text { through } \\
\text { HPM }\end{array}$ \\
\hline 0 & 0 & 0 & 0 & 0 \\
\hline 0.1 & 0.051151485 & 0.049261 & 0.0484625 & 0.221091 \\
\hline 0.2 & 0.098508173 & 0.095452 & 0.1353969 & 0.360656 \\
\hline 0.3 & 0.141875575 & 0.13853 & 0.1732599 & 0.449731 \\
\hline 0.4 & 0.181006393 & 0.178066 & 0.2068777 & 0.508866 \\
\hline 0.5 & 0.215581171 & 0.213394 & 0.2357687 & 0.55069 \\
\hline 0.6 & 0.245183718 & 0.243738 & 0.2593566 & 0.582128 \\
\hline 0.7 & 0.269277136 & 0.268316 & 0.2769773 & 0.606284 \\
\hline 0.8 & 0.287206039 & 0.286429 & 0.2879372 & 0.623979 \\
\hline 0.9 & 0.298285213 & 0.297529 & 0.2916666 & 0.634942 \\
\hline 1 & 0.302021673 & 0.301269 & & 0.638672 \\
\hline
\end{tabular}

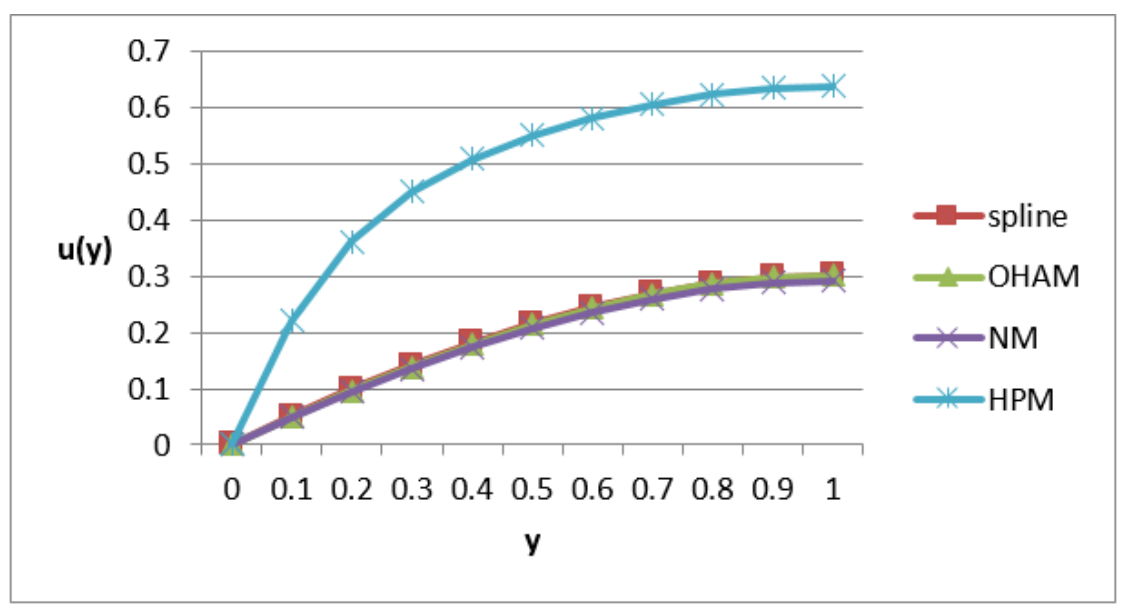

Figure 2. Comparison of spline collocation with OHAM, NM and HPM for $\beta=1.4, \mathrm{~m}=0.7$

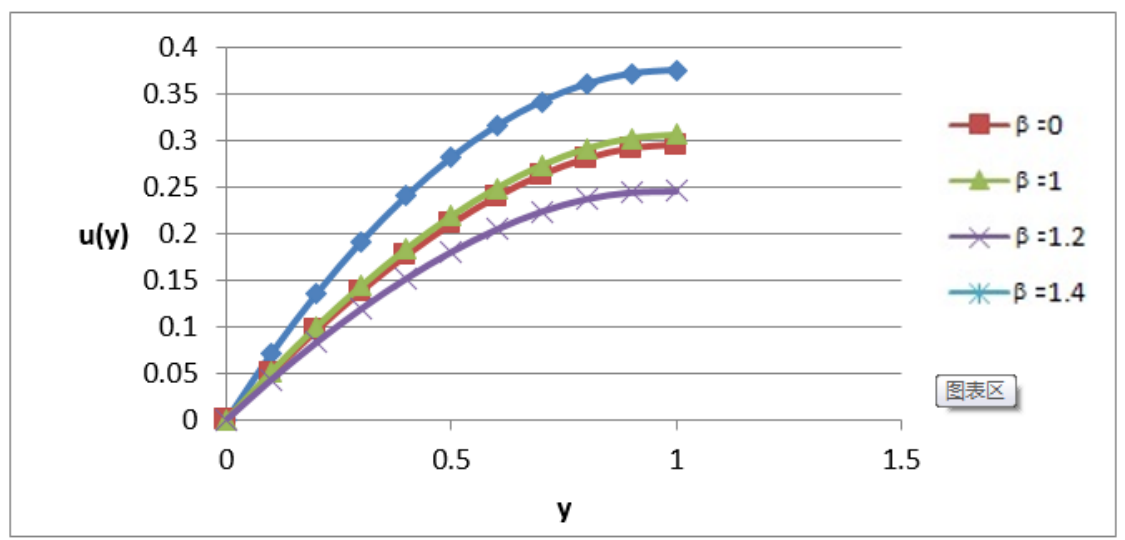

Figure 3. Effects on velocity profile for various values of $\beta$ at $m=0: 75$ 


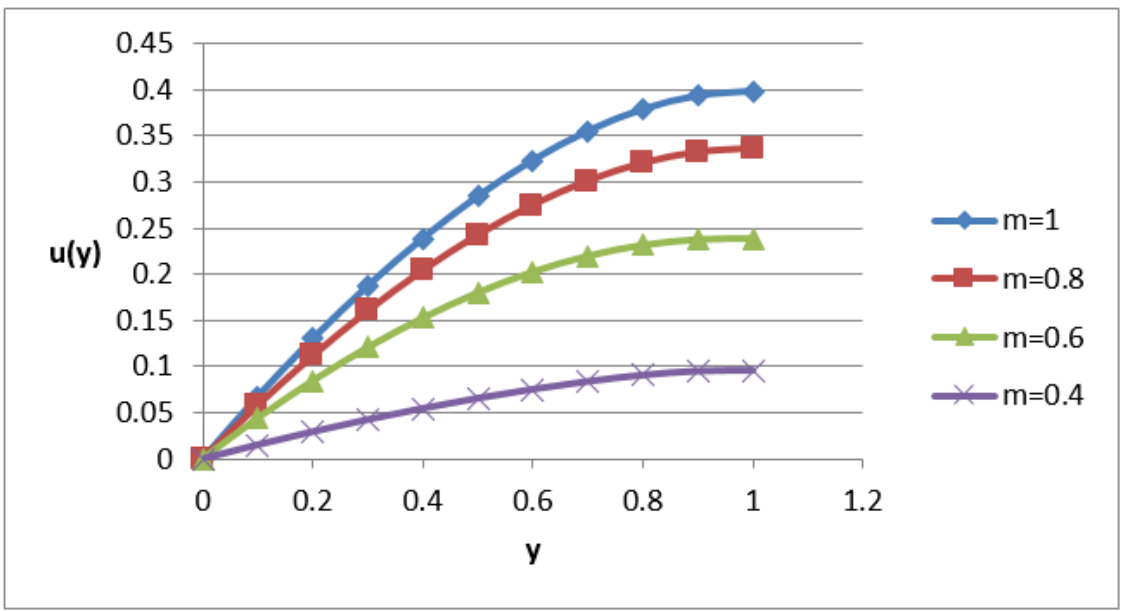

Figure 4. Effects on velocity profile for various values of $\mathrm{m}$ at $\beta=0.5$

\section{$5 \quad$ Results and Discussion}

The effects of controlling parameters on the velocity profile are presented by graphs in this work. The accuracy of the results are validated in table 1 and sketched by graph in figure 2 for $\mathrm{m}=0.75$ and $\beta=1.4$. It is clear that the spline method gives lowest error as compared to HPM, which is mentioned in figure 2.

Figure 3 indicates that for increasing values of parameters $\beta$ and for fixed value of $\mathrm{m}$ a velocity decreases, whereas if $\beta$ is kept fixed and $m$ decreases, then the velocity decreases, which is clear from figure 4 .

\section{Conclusion}

In the present paper, we have analyzed a thin film flow of third grade fluid down an inclined plane. The approximate solutions are obtained using spline collocation method for the nonlinear problem. The comparison results are shown in table as well as by graphs. Also the results are sketched for the fluid parameters $\beta$ and $\mathrm{m}$. Lastly we conclude that the spline collocation method provides a better approximation to the strong nonlinear fluid problems.

Acknowledgements. The authors would like to thank Prof. Dr. Harish D. and Doctor, Professor \& Head, Veer Narmad, South Gujarat University, Surat, Gujarat, India, for their helpful suggestions.

\section{References}

1. He.J.H." Homotopy perturbation method for solving boundary value problems" Phys.Lett.A.350,87-98. (2006).

2. He.J.H. "Non-perturbative methods for strongly non-linear problems Berlin Dissertation". De-verlagim Internet GrmbH. (2006)

3. Liao, Sh J. "Beyond perturbation: Introduction to the homotopy analysis method. 2003." Champan Hall, CRC, Boca Raton.

4. Marinca, Vasile, and Nicolae Herişanu. "Application of optimal homotopy asymptotic method for solving nonlinear equations arising in heat transfer." International Communications in Heat and Mass Transfer 35.6 (2008): 710-715.

5. Fazle Mabood "Comparison of optimal homotopy asymptotic method and homotopy perturbation method for strongly non-linear method" Journal of the Association of Arab Universities for Basic and Applied Sciences. (2014)16,21-26. 
6. Itishree Nayak,Ajit Kumar Nayak and Sudarsan Padhy "Numerical Solution for the Flow and Heat Transfer of a Third Grade Fluid Past a Porous Vertical Plate" Adv. Studies Theor. Phys., Vol 6,2012, no.13,615-624.

7. Jigisha U. Pandya and Harish D. Doctor." The Numerical Solution of Föppl-Hencky Equation Using Spline Method." Int. J. of Appl. Math and Mech. 6 (x): xx, 2010

8. Blue J L (1969) Spline function methods for nonlinear boundary value problems, Communications of the ACM, 12(6), 327-330 